\section{Yield and Fruit Quality of Sixteen Fragaria vesca Accessions Grown in Southern Florida}

\author{
Alan Chambers ${ }^{1}$, Pamela Moon, Yuqing Fu, and Juliette Choiseul
}

Tropical Research and Education Center, Horticultural Sciences Department, University of Florida, 18905 SW 280th Street, Homestead, FL 33031

Jinhe Bai, Anne Plotto, and Elizabeth Baldwin
Horticultural Research Laboratory, USDA-ARS, 2001 South Rock Road, Ft.
Pierce, FL 34945

Additional index words. acidity, aroma, total soluble solids, winter production

\begin{abstract}
Fragaria vesca is a diploid strawberry species that produces gourmet, aromatic fruits with only limited commercial production because of its relative obscurity. Most $F$. vesca research focuses on genetics and fruit aroma, but yield and fruit quality data across diploid accessions are lacking. Sixteen $F$. vesca accessions were grown in replicated field plots in southern Florida to measure field performance and fruit quality over multiple harvests during a single growing season. Accessions 'Reine des Vallees', 'Baron Solemacher', 'Fragolina di Bosco', and 'Reugen' all had significantly higher yield (115-140 g/plot/week) and fruit number (117-139 fruit/plot/week) compared with 'Bowlenzauber', 'Attila', 'Ali Baba', and 'Pineapple Crush' (31-57 g/plot/week and 32-60 fruit/plot/week) during peak production. Total average yield ranged from $240 \mathrm{~g}$ ('Pineapple Crush') to $1194 \mathrm{~g}$ ('Baron Solemacher') per plot of 10 plants. Fruit number and fruit yield were highly correlated $\left(R^{2}=0.96\right)$ for all accessions, and there was no significant difference in fruit weight among accessions through the entire season. Total soluble solids ranged from 10.9 to $13.5{ }^{\circ}$ Brix, and fructose, glucose, sucrose, and total sugars ranged from 15.3 to $22.1,13.5$ to $20.0,0.1$ to 2.7 , and 29.7 to $42.5 \mathrm{mg} / \mathrm{g}$, respectively, fresh weight. Acidity ranged from $1.00 \%$ to $1.18 \%$ citric acid and was not consistently significantly different among accessions over multiple harvests. Forty-two aroma compounds were putatively identified over three harvests for each accession and included mostly esters and ketones with a few alcohols, terpenes, and aldehydes. The majority of these compounds were similarly abundant over harvests and among accessions with a few exceptions, including methyl anthranilate. These results are the first in-depth study of yield and fruit quality for a large number of $F$. vesca accessions that could lead to increased cultivation of this species for local markets.
\end{abstract}

Strawberries are widely known for their attractive, sweet, and aromatic fruits. Global strawberry production was approximately 9.1 million metric tons in 2016, with California and Florida making up the majority of U.S. production (U.S. Department of Agriculture 2016; FAO 2016). Strawberry cultivation in Florida is concentrated in the west-central section of the state and significantly contributes to commercial strawberry production. The subtropical climate of this area favors production of octoploid, Fragaria $\times$ ananassa strawberries, from December to March (MacKenzie et al., 2011). Cultivars specifically bred for this location have demonstrated high yield, disease resistance, and fruit quality (Whitaker et al., 2011). In contrast, strawberry cultivation in southern Florida is

Received for publication 21 June 2018. Accepted for publication 3 Aug. 2018.

We thank David R. Wood for validating the Atago Pal-Easy ACID F5 by comparing with the Metrohm titrator for strawberries.

${ }^{1}$ Corresponding author. E-mail: ac@uff.edu. limited in part by high land prices caused by the proximity to major urban areas. Growers maintain economic viability by targeting premium, local markets, and by providing onfarm experiences. The fruit quality attributes of $F$. vesca are unique compared with those of $F$. Xananassa strawberries (Ulrich et al., 2007 ) and could provide opportunities for $F$. vesca cultivation on a larger scale. The $F$. vesca strawberries are small, soft, highly aromatic, and have an intense flavor. The plants are ever-bearing and morphologically distinct from commercial strawberries. Winter production of $F$. vesca could provide an opportunity for local growers to move into premium markets by producing noncommodity, gourmet strawberries as part of their existing operations.

$F$. vesca strawberries are also known as alpine strawberries, diploid strawberries, or gourmet strawberries. $F$. vesca has been an attractive model organism for functional genomics research for Rosaceae species, including commercial strawberry, because of its small genome size, compact growth habit, rapid generation cycle, published genome, and efficient transformation protocols (Hadonou et al., 2004; Hirakawa et al., 2014; Oosumi et al., 2006; Sargent et al., 2004; Shulaev et al., 2011). Despite having a global geographic range, diploid strawberries have limited cultivation because of poor shipping quality and are typically only available for local markets (Doumett et al., 2011; Nin et al., 2017).

Many named $F$. vesca varieties exist, including both red and yellow fruit types. Fruit quality differences between diploid and octoploid strawberries have been investigated by many groups. Aroma profile differences between diploid and octoploid strawberries (Dong et al., 2013; Ulrich et al., 1997, 2007), between diploid and the hexaploid $F$. moschata (Negri et al., 2015), and among $F$. vesca accessions (Ulrich and Olbricht, 2013) have been reported. Differences in nutraceutical content among $F$. vesca accessions (Giordani et al., 2016), and as contrasted to octoploid strawberries (Crespo et al., 2010; Doumett et al., 2011), also have been reported. Much of this foundational work lends itself to further genetic and biochemical research (Ulrich and Olbricht 2014). This is also valuable information, when combined with sensory analysis, to identify and select accessions with superior flavor.

Although $F$. vesca strawberries are known for their favorable aroma, information on their yield performance in open-field production is lacking. F. vesca cultivation could be economically viable in southern Florida for niche and specialty markets. Previous research reported yield and fruit quality data for four accessions at two locations over a single season in Switzerland (Crespo et al., 2010 ) and for one $F$. vesca cultivar under hydroponic cultivation in Italy (Caruso et al., 2011; Nin et al., 2017). This type of information is vital to understanding the economics of growing $F$. vesca for various markets.

The objectives of this study were to measure yield and fruit quality differences among a collection of $F$. vesca accessions and to identify superior accessions grown during the winter production season in southern Florida. These results reduce the risk of adopting $F$. vesca as a high-value specialty crop, especially in southern Florida, by identifying the highest yielding and highestquality accessions for grower and homeowner cultivation of this crop.

\section{Materials and Methods}

Plant materials. All $F$. vesca accessions used in this study were either obtained as seeds from the U.S. Department of Agriculture, germplasm repository in Corvallis, OR (as annotated by PI accession numbers in parentheses to follow), or through online vendors. Accessions selected for this study included red-fruited types 'Alexandria' (PI 602923), 'Alba' (PI 616917), 'Ali Baba', 'Attila', 'Baron Solemacher' (PI 551507), 'Bowlenzauber', 'Fragolina di Bosco', 
'Mignonette' (PI 616935), 'Quattro Stagioni', 'Reine des Vallees' (PI 551824), 'Reugen' (PI 551834), and 'Semperflorens' ( $F$. vesca L. subsp. vesca f. semperflorens, PI 552288), and yellow-fruited types 'Golden Alpine' (PI 616576), 'Ivory', 'White Delight', and 'White Solemacher',

Plant growth. Strawberry seeds were sown directly in soilless mix augmented with slow-release fertilizer and grown in a greenhouse. Individual plants were maintained in 38-cell flats for 3 months until transplanting to the field in December during the 2016-17 Winter growing season. While in the greenhouse, flats were fertilized monthly with $0.5 \times$ Hoagland solution. The field planting was a complete randomized block design with 10 plants per plot and three plots per accession. Plants were established with $38-\mathrm{cm}$ spacing in two rows on mounded, plastic mulch-covered beds. Plants were fertilized daily with $3-0-10$ (N-P-K) through drip irrigation during watering. One application of metaldehyde (2,4,6,8-tetramethyl- 1,3,5,7-tetraoxycyclooctane, Deadline ${ }^{\circledR}$ M-Ps; Amvac, Los Angeles, CA) was necessary to control snails at the beginning of the season. No other chemical treatments were applied to the field planting.

Yield. Strawberries were harvested twice each week starting on 19 Jan. 2017 (harvest week 1) when the first fruit were ripe and continued for 12 weeks until early 3 Apr. 2017 (harvest week 12). Fruit number and total weight data were collected by plot. Yield data were collected within $4 \mathrm{~h}$ of harvest. Total average fruit yield was calculated from three plots of 10 plants each.

Soluble solids. Total soluble solids data were generated by blending $\approx 20 \mathrm{~g}$ of fruit from individual plots for each harvest at weeks 10,11, and 12. The homogenized sample was then centrifuged at $3200 \mathrm{~g}$ for 30 min to obtain a cleared supernatant. The supernatant was applied directly to the sensor of an Atago Pal-1 digital pocket refractometer according to the manufacturer's instructions (Atago USA, Bellevue, WA). Three replicates from each plot were processed resulting in nine replicate samples per accession per harvest. Total soluble solids data are reported as ${ }^{\circ}$ Brix.

High-performance liquid chromatography (HPLC) sugar quantification. Individual sugars (sucrose, glucose, and fructose) were analyzed with an HPLC system (HPLC pump and Auto sampler, Series 200; Perkin Elmer, Waltham, MA) and their values summed for total sugars. Twenty-five grams of fruit per accession were homogenized using a blender. Ten grams of each homogenate were then diluted $50 \%$ with deionized water, centrifuged (Avanti J-E centrifuge; Beckman-Coulter, Brea, CA) at 11,952g, and the supernatant was filtered through a $0.45-\mu \mathrm{m}$ Millipore (Siemens-Millipore, Shrewbury, MA) filter before analysis by HPLC with a Sugar-Pak column $(10 \mu \mathrm{m}, 6.5 \mathrm{~mm} \times 300 \mathrm{~mm}$; Waters, Milford, MA) operated at $90{ }^{\circ} \mathrm{C}$ in a $\mathrm{CH}-30$ column heater and a TC-50 controller (FiAtron, Milwaukee, WI) using an Agilent 1260 series refractive index detector (Agilent Technologies, Santa Clara, CA). Quantification was based on the external standard method (EZChrom Elite software, Version 3.3.2., SP2; Agilent Technologies) using standards for sucrose, glucose, and fructose and adjusted for the sample dilution. All results are expressed as milligram per gram of fresh fruit (Baldwin et al., 2012). Two replicates per accession were analyzed using combined samples from multiple harvests.

Acidity. Acidity was measured using an Atago Pal-Easy ACID F5 on the strawberry setting (Atago USA). Samples from individual plots for each harvest at weeks 10,11, and 12 were homogenized and centrifuged at $3200 \mathrm{~g}$ for $30 \mathrm{~min}$ as for soluble solids, except the cleared supernatant was diluted 1:50 in distilled water before measurement according to the manufacturer's instructions. Three replicates from each plot were processed resulting in nine replicate samples per accession per harvest. Acidity data are reported as percent citric acid on a fresh weight basis. Preliminary research showed good correlation between titratable acidity measured by titration with $0.1 \mathrm{~mol} \cdot \mathrm{L}^{-1} \mathrm{NaOH}$ to $\mathrm{pH} 8.1$ using a Metrohm titrator (Riverview, FL) and titratable acidity measured with the Atago PalEasy ACID F5 (A. Plotto, unpublished data).

Volatiles analysis. Volatiles were analyzed using a headspace gas chromatography mass spectrometry system as previously reported (Chambers et al., 2014). To summarize, fruit for aroma analysis were harvested on 7, 14, and 21 Apr. (harvests 12, 13, and 14). All fruit used for aroma analysis were clean and blemish-free. Representative 40-g fruit samples were homogenized with an equal weight of saturated $\mathrm{NaCl}(\approx 35 \mathrm{~g} / \mathrm{L}$ $\mathrm{NaCl}$ in distilled water) and 3-hexanone as an internal standard at $1 \mathrm{ppm}$. Two $5-\mathrm{mL}$ aliquots representing two technical replicates for each sample were pipetted into $20-\mathrm{mL}$ glass Gerstel vials and sealed with magnetic crimp caps (Gerstel, Baltimore, MD). A 2-cm triphase solid-phase microextraction (SPME) fiber $\quad(50 / 30 \mu \mathrm{m}$ DVB/Carboxen/PDMS; Supelco, Bellefonte, PA) was used to collect and concentrate volatiles before running on a DB-5 column $(60 \mathrm{~m} \times 0.25 \mathrm{~mm}$ i.d., $1.00-\mu \mathrm{m}$ film thickness, J\&W Scientific, Folsom, CA) equipped Agilent 6890 gas chromatograph (GC) coupled with a $5973 \mathrm{~N}$ mass spectrometer (MS) detector (Agilent Technologies, Palo Alto, CA). Before analysis, samples were held at $4{ }^{\circ} \mathrm{C}$ in a Peltier cooling tray attached to a MPS2 autosampler (Gerstel, Baltimore, MD). All other volatile sampling and analysis methods were as previously described (Jouquand et al., 2008). All volatile concentrations were normalized to the internal standard and reported as ng/g.

Statistics. All figures and statistical analyses were created and conducted using JMP Pro v13 (SAS Institute, Cary, NC). Tukey's honestly significant difference $(\alpha \leq 0.05)$ was used for mean separation. Hierarchical clustering with Ward distance for aroma analysis also was conducted using JMP.

\section{Results}

Yield. Total weekly fruit weight for each accession over the growing season showed significant differences (Fig. 1). 'Baron Solemacher' was the first accession to produce fruit, starting 19 Jan. 2017. Most accessions reached peak yield during weeks 9,10 , and 11 (13, 20, and 27 Mar.), with a general decline in yield from week 11 to 12 . The average weekly plot fruit weight (10 plants/ plot) during weeks 9 to 11 of 'Reine des Vallees' (140.5 g), 'Baron Solemacher' (132.6 g), 'Fragolina di Bosco' (130.8 g), 'Reugen' (115.3 g), and 'White Delight' $(105.7 \mathrm{~g})$ were significantly higher than 'Golden Alpine' (65.8 g), 'Quattro Stagioni' (64.3 g), 'Bowlenzauber' (57.1 g), 'Attila' (49.7 g), 'Ali Baba' (34.1 g), and 'Pineapple Crush' (31.6 g) (Table 1).

The average plot fruit number from weeks 9 to 11 also was considered as a yield component (Fig. 2; Table 1). The average number of fruit per 10-plant plot for weeks 9 to 11 for 'Fragolina di Bosco' (139.7), 'Baron Solemacher' (125.1), 'Reugen' (120.8), and 'Reine des Vallees' (117.6) was significantly higher than that of 'Quattro Stagioni' (64.4), 'Golden Alpine' (61.7), 'Bowlenzauber' (59.8), 'Attila' (48.3), ‘Ali Baba' (39.8), and 'Pineapple Crush' (32.8).

Total average plot yield ranged from the highest two accessions at $1194.5 \mathrm{~g}$ and $1180.1 \mathrm{~g}$ for 'Baron Solemachaer' and 'Reine des Vallees', respectively, to the lowest yield for accessions at $242.9 \mathrm{~g}$ and $240.6 \mathrm{~g}$ for 'Ali Baba' and 'Pineapple Crush', respectively. 'Baron Solemacher', 'Reine des Vallees', 'Fragolina di Bosco', 'Reugen', and 'Semperflorens' all had significantly higher total average plot yield than 'Ali Baba' and 'Pineapple Crush'.

Average plot yield and fruit number across the entire season for all accessions were highly correlated at $R^{2}=0.96$, and there was no significant difference in average fruit weight among accessions. The average fruit weight across the entire season for all accessions was therefore calculated at $1.15 \mathrm{~g}$ under these growing conditions.

Total soluble solids (TSS). TSS were quantified at harvest weeks 10,11 , and 12 (20 and 27 Mar. and 3 Apr.) for all 16 accessions (Table 2). These harvest weeks overlapped with peak yield. Average TSS for all three harvests ranged from a high of 13.37 ('Ali Baba') to 10.94 ('Reine des Vallees') ${ }^{\circ}$ Brix. Only 'Ali Baba' (13.37) and 'Mignonette' (12.10) had significantly higher TSS than the lowest accessions 'Pineapple Crush' (11.15) and 'Reine des Vallees' (10.94).

HPLC sugar quantification. Fruit samples from harvests 9,10 , and 11 were bulked for each accession and analyzed for sugar content by HPLC (Table 1). Fructose ranged from 15.6 to $21.9 \mathrm{mg} / \mathrm{g}$ fresh weight $(\mathrm{FW})$, glucose from 14.1 to $19.5 \mathrm{mg} / \mathrm{g} \mathrm{FW}$, sucrose from 0.10 to $2.2 \mathrm{mg} / \mathrm{g} \mathrm{FW}$, and total sugars from 29.9 to $42.4 \mathrm{mg} / \mathrm{g}$ FW. 'Mignonette', 'White Delight', 'Baron Solemacher', and 'Ivory' all had significantly higher total sugar content than 'Golden Alpine', 'Semperflorens', and 'Attila'. Total sugar content correlated well with percent fructose $\left(R^{2}=0.95\right)$ and percent glucose $\left(R^{2}=0.96\right)$ but not with percent sucrose $\left(R^{2}=0.03\right)$ or TSS $\left(R^{2}=0.09\right)$. 


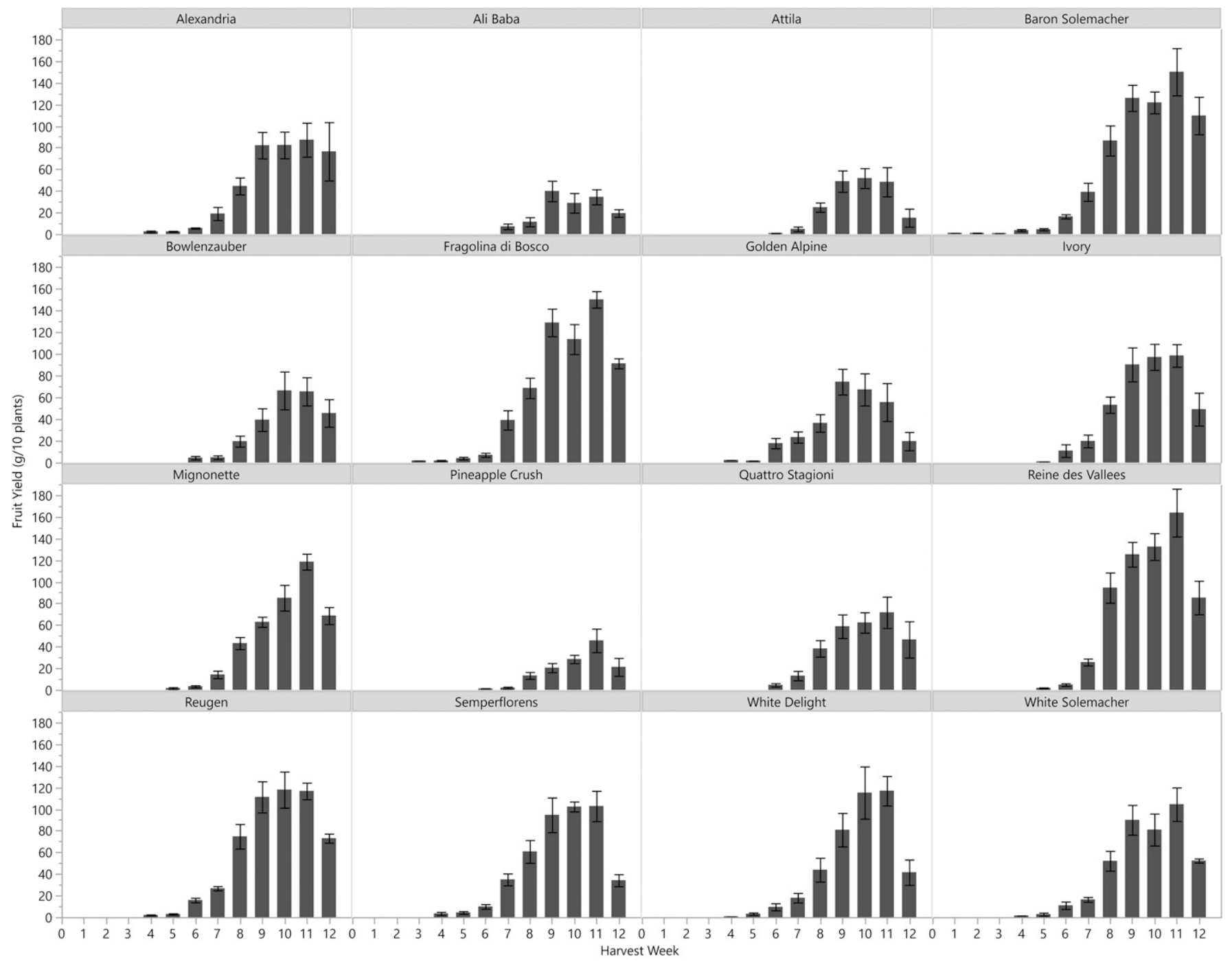

Fig. 1. Total yield of all Fragaria vesca accessions included in this study. Yield data are shown as grams fresh weight by harvest week starting 19 Jan. 2017 , when the earliest accession produced ripe berries. Data for each accession are averaged from three plots of 10 plants each. Bars represent the SE.

Table 1. Yield and fruit quality traits for 16 Fragaria vesca accessions. $^{\mathrm{z}}$

\begin{tabular}{|c|c|c|c|c|c|c|c|}
\hline Accession & $\begin{array}{l}\text { Avg plot } \\
\text { yield }\end{array}$ & $\begin{array}{c}\text { Avg plot } \\
\text { fruit number }\end{array}$ & $\begin{array}{l}\text { Avg total } \\
\text { plot yield }\end{array}$ & $\begin{array}{c}\text { Fructose } \\
\text { (mg/g FW) }\end{array}$ & $\begin{array}{c}\text { Glucose } \\
(\mathrm{mg} / \mathrm{g} \mathrm{FW})\end{array}$ & $\begin{array}{c}\text { Sucrose } \\
(\mathrm{mg} / \mathrm{g} \mathrm{FW})\end{array}$ & $\begin{array}{l}\text { Total sugars } \\
(\mathrm{mg} / \mathrm{g} \mathrm{FW})\end{array}$ \\
\hline Ali Baba & $34.1(\mathrm{H})$ & $39.8(\mathrm{G})$ & 242.9 (E) & $20.6(\mathrm{~A}-\mathrm{C})$ & $18.5(\mathrm{AB})$ & 0.2 (B) & $39.3(\mathrm{~A}-\mathrm{C})$ \\
\hline Attila & $49.7(\mathrm{GH})$ & 48.3 (FG) & $362.5(\mathrm{DE})$ & $15.6(\mathrm{I})$ & $14.1(\mathrm{C})$ & $0.2(\mathrm{~B})$ & 29.9 (E) \\
\hline Bowlenzauber & $57.1(\mathrm{~F}-\mathrm{H})$ & 59.8 (D-G) & 436.7 (C-E) & 18.7 (D-F) & $17.5(\mathrm{~A}-\mathrm{C})$ & $2.2(\mathrm{~A})$ & $38.4(\mathrm{~A}-\mathrm{D})$ \\
\hline Fragolina di Bosco & $130.8(\mathrm{~A}-\mathrm{C})$ & $139.7(\mathrm{~A})$ & $1110.0(\mathrm{AB})$ & $18.2(\mathrm{D}-\mathrm{H})$ & $17.2(\mathrm{~A}-\mathrm{C})$ & 0.7 (B) & 36.1 (B-D) \\
\hline Golden Alpine & $65.8(\mathrm{E}-\mathrm{H})$ & $61.7(\mathrm{E}-\mathrm{G})$ & 558.5 (B-E) & $17.0(\mathrm{H})$ & $15.7(\mathrm{~A}-\mathrm{C})$ & $1.1(\mathrm{AB})$ & $33.8(\mathrm{DE})$ \\
\hline Pineapple Crush & $31.6(\mathrm{H})$ & $32.8(\mathrm{G})$ & $240.6(\mathrm{E})$ & $17.5(\mathrm{~F}-\mathrm{H})$ & $15.8(\mathrm{~A}-\mathrm{C})$ & $1.3(\mathrm{AB})$ & 34.6 (C-E) \\
\hline Quattro Stagioni & $64.3(\mathrm{E}-\mathrm{H})$ & $64.4(\mathrm{D}-\mathrm{G})$ & 541.5 (B-E) & 18.8 (D-F) & $17.0(\mathrm{~A}-\mathrm{C})$ & 0.1 (B) & 35.9 (B-D) \\
\hline Reine des Vallees & 140.5 (A) & $117.6(\mathrm{~A}-\mathrm{C})$ & $1180.1(\mathrm{~A})$ & $17.9(\mathrm{E}-\mathrm{H})$ & $16.6(\mathrm{~A}-\mathrm{C})$ & $0.2(\mathrm{~B})$ & 34.7 (C-E) \\
\hline Reugen & 115.3 (A-D) & $120.8(\mathrm{AB})$ & 1005.7 (A-C) & $18.8(\mathrm{DE})$ & $17.9(\mathrm{~A}-\mathrm{C})$ & 0.7 (B) & 37.4 (B-D) \\
\hline Semperflorens & 99.6 (B-E) & 98.5 (B-D) & 874.9 (A-D) & $17.4(\mathrm{GH})$ & $15.5(\mathrm{BC})$ & $0.8(\mathrm{~B})$ & $33.7(\mathrm{DE})$ \\
\hline White Delight & 105.7 (A-D) & 99.6 (B-D) & 769.3 (A-E) & $21.0(\mathrm{AB})$ & 19.4 (A) & $0.2(\mathrm{~B})$ & $40.6(\mathrm{AB})$ \\
\hline White Solemacher & 91.8 (D-F) & 82.9 (C-F) & $754.0(\mathrm{~A}-\mathrm{E})$ & $19.4(\mathrm{CD})$ & $17.6(\mathrm{~A}-\mathrm{C})$ & $0.6(\mathrm{~B})$ & $37.6(\mathrm{~A}-\mathrm{D})$ \\
\hline
\end{tabular}

${ }^{\mathrm{z}}$ Average weekly plot yield (10 plants/plot) and fruit number are from weeks 9 to 11, which was the time of peak fruit production. Quantification of fructose, glucose, sucrose, and total sugars (mg/g FW) are from fruit bulked across these three harvest weeks. Results denoted with the same connecting letters in parentheses are not significantly different at the $\alpha \leq 0.05$ level using the Tukey's honestly significant difference multiple comparison test. $\mathrm{FW}=$ fresh weight.

Acidity. Acidity was quantified for each accession during the same harvests as reported for TSS (weeks 10, 11, and 12 on 20 and 27 Mar. and 3 Apr., respectively) using the preprogrammed strawberry setting on an Atago Pal-Easy ACID F5 (Table 2). Only the highest average acidity for 'Semperflorens' (1.18\% citric acid) was signifi- cantly different from the lowest acidity level of all accessions (1.00\% citric acid, 'Reine des Vallees') when averaged across all harvests. 


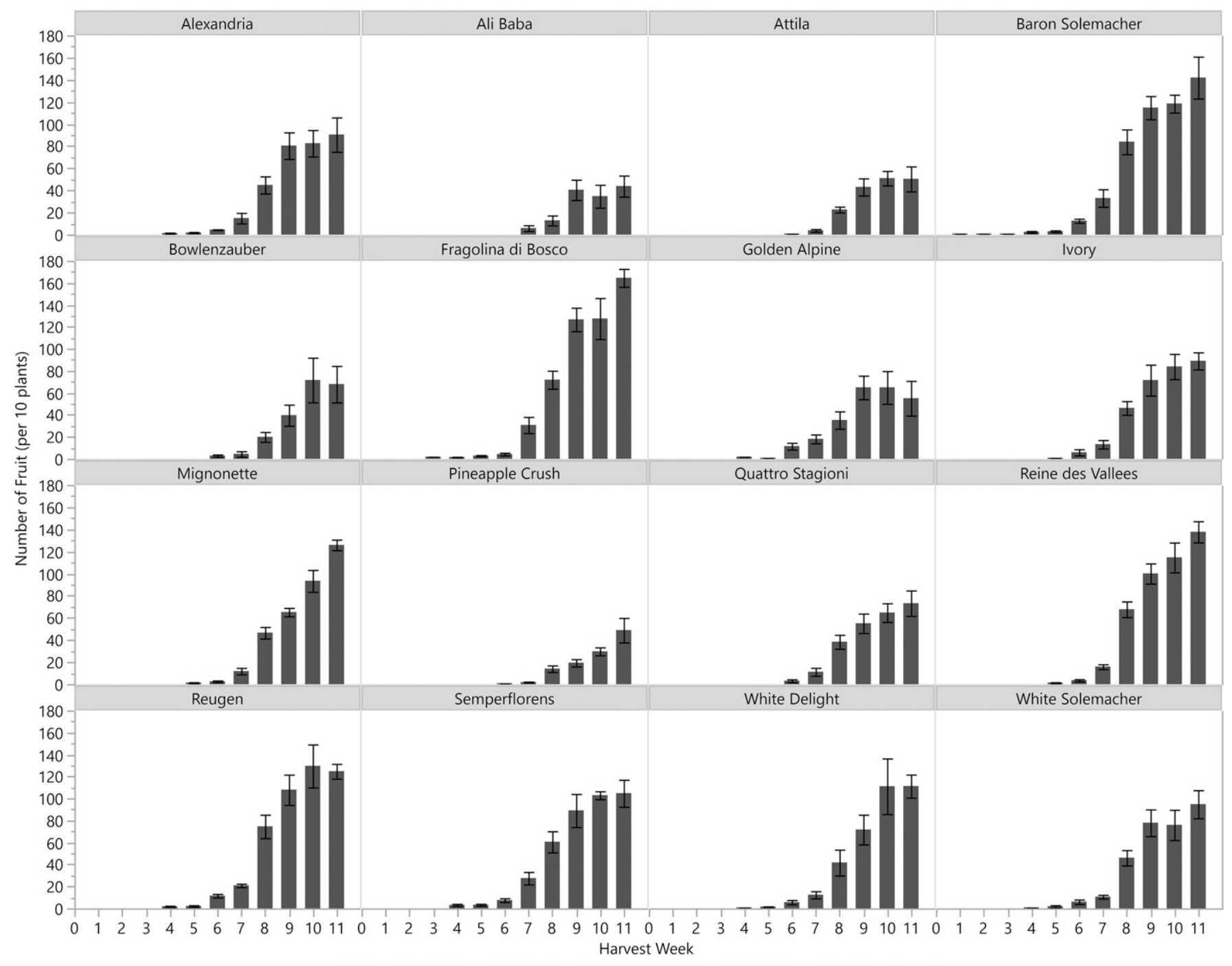

Fig. 2. Total number of fruit from all Fragaria vesca accessions included in this study. Fruit number data are shown by harvest week starting when the earliest accession produced ripe berries. Data for each accession are from three plots of 10 plants each. Bars represent the SE.

Table 2. Average total soluble solids (TSS) and acidity (\% citric acid) for each accession at each of three harvests. ${ }^{2}$

\begin{tabular}{|c|c|c|c|c|c|c|c|c|}
\hline \multirow[b]{2}{*}{ Accession } & \multicolumn{4}{|c|}{ TSS } & \multicolumn{4}{|c|}{ Acidity } \\
\hline & Harvest 10 & Harvest 11 & Harvest 12 & Total Avg & Harvest 10 & Harvest 11 & Harvest 12 & Total avg \\
\hline Ali Baba & $15.11(0.37)$ & $12.86(0.30)$ & $12.53(0.64)$ & $13.37(0.29, \mathrm{~A})$ & $1.39(0.07)$ & $1.07(0.07)$ & $1.07(0.04)$ & $1.15(0.05, \mathrm{AB})$ \\
\hline Bowlenzauber & $11.57(0.66)$ & $11.58(0.21)$ & $12.50(0.60)$ & $11.71(0.24, \mathrm{~B}-\mathrm{D})$ & $1.19(0.06)$ & $1.16(0.05)$ & $1.04(0.05)$ & $1.15(0.04, \mathrm{AB})$ \\
\hline Fragolina di Bosco & $12.00(0.22)$ & $11.48(0.30)$ & $11.23(0.37)$ & $11.62(0.19, \mathrm{~B}-\mathrm{D})$ & $1.11(0.04)$ & $1.05(0.05)$ & $1.04(0.05)$ & $1.07(0.03, \mathrm{AB})$ \\
\hline Golden Alpine & $12.31(0.43)$ & $11.81(0.26)$ & $12.30(0.78)$ & $12.01(0.21, \mathrm{BC})$ & $1.09(0.08)$ & $1.01(0.03)$ & $1.08(0.11)$ & $1.04(0.03, \mathrm{AB})$ \\
\hline Pineapple Crush & $11.32(0.30)$ & $11.10(0.21)$ & $10.80(0.80)$ & $11.15(0.16, \mathrm{CD})$ & $1.14(0.04)$ & $1.09(0.05)$ & $1.18(0.04)$ & $1.11(0.03, \mathrm{AB})$ \\
\hline Quattro Stagioni & $12.34(0.20)$ & $11.80(0.23)$ & $10.97(0.98)$ & $11.88(0.18, \mathrm{BC})$ & $1.06(0.05)$ & $1.05(0.05)$ & $0.91(0.08)$ & $1.04(0.03, \mathrm{AB})$ \\
\hline Reine des Vallees & $11.28(0.21)$ & $10.73(0.20)$ & $11.17(0.77)$ & $10.94(0.15, \mathrm{D})$ & $0.98(0.04)$ & $1.01(0.03)$ & $1.01(0.07)$ & $1.00(0.02, \mathrm{~B})$ \\
\hline Reugen & $12.11(0.21)$ & $11.38(0.27)$ & $11.40(0.96)$ & $11.62(0.20, \mathrm{~B}-\mathrm{D})$ & $1.15(0.07)$ & $0.99(0.06)$ & $1.05(0.07)$ & $1.05(0.04, \mathrm{AB})$ \\
\hline Semperflorens & $11.76(0.23)$ & $11.81(0.15)$ & $11.60(0.45)$ & 11.77 (0.12, B-D) & $1.38(0.04)$ & $1.10(0.05)$ & $1.15(0.04)$ & $1.18(0.04, \mathrm{~A})$ \\
\hline White Delight & $11.91(0.34)$ & $11.12(0.17)$ & $12.10(0.86)$ & $11.45(0.17, \mathrm{~B}-\mathrm{D})$ & $1.20(0.05)$ & $1.03(0.04)$ & $1.04(0.10)$ & $1.08(0.03, \mathrm{AB})$ \\
\hline White Solemacher & $11.91(0.23)$ & $11.76(0.21)$ & $12.20(0.56)$ & $11.84(0.15, \mathrm{BC})$ & $1.07(0.08)$ & $1.07(0.04)$ & $1.28(0.05)$ & $1.09(0.03, \mathrm{AB})$ \\
\hline
\end{tabular}

${ }^{\mathrm{z}}$ Total average TSS and total average acidity are average values for each genotype for all three harvests. Average values are from three replicates of 10 plant plots. SE is shown in parentheses. Tukey's honestly significant difference is also shown in parentheses as connecting letters where accessions with the same connecting letter at not significantly different at $\alpha \leq 0.05$.

Aroma analysis. In all, 42 aroma compounds were detected and putatively identified using SPME and GC-MS (Table 3). The total relative peak area of all 42 aroma compounds for each $F$. vesca accession for each harvest showed variability among accessions and over harvests (Fig. 3). Total relative peak area varied more than 2-fold for most accessions among harvests. Most aroma compounds were detected in every accession during at least one of the three harvest samplings. Greater than 


\begin{tabular}{|c|c|c|c|c|c|c|}
\hline \multirow[b]{2}{*}{ Compound } & \multirow[b]{2}{*}{ RT (min) } & \multirow[b]{2}{*}{ RI } & \multirow[b]{2}{*}{ Chemical class } & \multicolumn{3}{|c|}{ Relative peak area (range in $\mathrm{ng} / \mathrm{g}$ ) } \\
\hline & & & & Harvest 12 & Harvest 13 & Harvest 14 \\
\hline Unknown 1 & 3.17 & 500 & Other & $0.627-0.788$ & $0.805-1.187$ & $0.729-1.351$ \\
\hline 4-Methyl-2-pentanol & 4.36 & 534 & Alcohol & $0.087-0.359$ & $0.175-1.220$ & $0.152-1.179$ \\
\hline 2-Heptanone & 4.90 & 548 & Ketone & $0.092-0.378$ & $0.201-0.849$ & $0.200-0.887$ \\
\hline Unknown 8 & 5.50 & 565 & Other & $0.301-0.956$ & $0.235-0.538$ & $0.221-0.927$ \\
\hline Ethyl acetate & 7.47 & 617 & Ester (ethyl) of acetic acid & $1.697-5.451$ & $1.142-9.886$ & $1.267-9.441$ \\
\hline Methyl propanoate & 8.10 & 634 & Ester (methyl) & $0.019-0.057$ & $0.017-0.079$ & $0.009-0.048$ \\
\hline 2-Pentanone & 10.06 & 683 & Ketone & $0.000-0.791$ & $0.889-4.291$ & $0.473-4.562$ \\
\hline Ethyl propanoate & 10.91 & 704 & Ester (ethyl) & $0.110-0.237$ & $0.190-0.314$ & $0.153-0.382$ \\
\hline 5-Methyl-3-heptanone & 11.04 & 708 & Ketone & $0.147-0.307$ & $0.126-0.291$ & $0.159-0.307$ \\
\hline Methyl butanoate & 11.38 & 716 & Ester (methyl) & $0.387-1.764$ & $0.563-2.406$ & $0.279-1.135$ \\
\hline 2-Methyl-1-propyl acetate & 13.53 & 768 & Ester of acetic acid & $0.221-0.463$ & $0.185-0.787$ & $0.174-0.615$ \\
\hline Methyl pentanoate & 13.72 & 773 & Ester (methyl) & $0.033-0.095$ & $0.013-0.086$ & $0.011-0.037$ \\
\hline Ethyl butanoate & 14.72 & 796 & Ester (ethyl) & $2.491-6.704$ & $5.099-10.921$ & $3.297-10.958$ \\
\hline Butyl acetate & 15.29 & 810 & Ester of acetic acid & $1.285-4.533$ & $2.420-4.891$ & $1.328-4.710$ \\
\hline Menthyl lactate & 17.04 & 851 & Ester & $0.033-0.097$ & $0.032-0.082$ & $0.040-0.122$ \\
\hline $2 E$-Hexenal & 17.38 & 858 & Aldehyde & $1.153-2.748$ & $1.222-2.483$ & $1.520-3.626$ \\
\hline $2 E$-Hexenol & 17.73 & 866 & Alcohol & $0.120-0.370$ & $0.192-0.794$ & $0.126-0.860$ \\
\hline Methyl butyl acetate & 18.24 & 878 & Ester of acetic acid & $0.247-0.571$ & $0.272-0.586$ & $0.170-0.400$ \\
\hline Butyl butanoate & 19.12 & 898 & Ester & $0.030-0.177$ & $0.107-0.380$ & $0.063-0.366$ \\
\hline Amyl acetate & 19.82 & 914 & Ester of acetic acid & $0.059-0.115$ & $0.072-0.185$ & $0.070-0.166$ \\
\hline Methyl hexanoate & 20.33 & 926 & Ester (methyl) & $0.068-0.553$ & $0.376-1.861$ & $0.242-1.426$ \\
\hline$\alpha$-Pinene & 21.29 & 948 & Terpene & $0.072-0.246$ & $0.108-0.370$ & $0.076-0.266$ \\
\hline Unknown 2 & 22.18 & 968 & Other & $0.649-1.210$ & $0.995-1.973$ & $0.875-1.639$ \\
\hline Ethyl heptanoate & 23.59 & 1001 & Ester (ethyl) & $0.228-1.149$ & $1.776-9.106$ & $0.762-9.308$ \\
\hline $3 E$-Hexenyl acetate & 23.93 & 1009 & Ester of acetic acid & $0.275-0.995$ & $0.289-1.326$ & $0.389-1.502$ \\
\hline Hexyl acetate & 24.17 & 1014 & Ester of acetic acid & $1.494-4.298$ & $3.039-7.068$ & $2.365-6.692$ \\
\hline $2 E$-Hexenyl acetate & 24.26 & 1016 & Ester of acetic acid & $1.280-2.975$ & $1.508-5.117$ & $1.420-4.161$ \\
\hline 2-Heptyl acetate & 25.28 & 1040 & Ester of acetic acid & $0.000-0.073$ & $0.055-0.374$ & $0.030-0.644$ \\
\hline Limonene & 25.50 & 1045 & Terpene & $0.014-0.220$ & $0.006-0.107$ & $0.060-0.310$ \\
\hline Unknown 3 & 26.79 & 1075 & Other & $0.000-1.658$ & $0.000-3.075$ & $0.001-4.772$ \\
\hline 2-Nonanone & 27.75 & 1098 & Ketone & $0.012-2.040$ & $1.569-8.764$ & $0.870-9.878$ \\
\hline Unknown 4 & 28.63 & 1119 & Other & $0.233-0.572$ & $0.356-0.706$ & $0.340-0.741$ \\
\hline Methyl octanoate & 28.99 & 1127 & Ester (methyl) & $0.000-0.094$ & $0.078-0.424$ & $0.055-0.283$ \\
\hline Benzyl acetate & 31.45 & 1187 & Ester of acetic acid & $0.000-0.080$ & $0.006-0.384$ & $0.003-0.478$ \\
\hline 2-Methyl-heptyl propanoate & 31.68 & 1193 & Ester & $0.000-0.023$ & $0.009-0.098$ & $0.005-0.077$ \\
\hline n-Nonanyl acetate & 32.36 & 1210 & Ester of acetic acid & $0.000-0.823$ & $0.583-2.593$ & $0.381-3.163$ \\
\hline Unknown 5 & 35.07 & 1280 & Other & $0.126-0.487$ & $0.162-0.767$ & $0.183-0.587$ \\
\hline Pinocarvyl acetate & 36.17 & 1309 & Ester of acetic acid & $0.025-0.059$ & $0.039-0.107$ & $0.030-0.113$ \\
\hline Myrtenyl acetate & 37.13 & 1336 & Ester of acetic acid & $0.551-1.365$ & $0.853-2.220$ & $0.786-2.617$ \\
\hline Methyl anthranilate & 39.36 & 1398 & Ester (methyl) & $0.002-0.048$ & $0.005-0.161$ & $0.002-0.090$ \\
\hline Unknown 6 & 40.84 & 1442 & Other & $0.027-0.224$ & $0.038-0.481$ & $0.033-0.265$ \\
\hline Unknown 7 & 45.93 & 1604 & Other & $0.013-0.083$ & $0.018-0.435$ & $0.015-0.096$ \\
\hline
\end{tabular}

${ }^{\mathrm{z}}$ Putative compound identification, RT (in minutes), RI, chemical class, and range of relative peak areas during each harvest are shown.

$\mathrm{RT}=$ retention time; $\mathrm{RI}=$ retention index.

2-fold changes in individual aroma compound relative peak areas were detected for individual accessions from harvest to harvest and from accession to accession.

Methyl anthranilate, a key aroma volatile, showed large fluctuations over harvests for most accessions, although its abundance in the cultivar Baron Solemacher seemed to be more consistent over three harvests than for other accessions (Fig. 4A). The volatile Unknown 3 showed a unique presence/absence pattern of among all volatiles and was only detected in 'Alexandria', 'Baron Solemacher', 'Bowlenzauber', 'Fragolina di Bosco', 'Golden Alpine', and 'Mignonette' for at least two harvests each (Fig. 4B).

A cluster analysis was performed for volatile data by accession and harvest (Fig. 5). The results are shown for each accession at three harvests and are colorcoded by harvest. The accessions from the 7 Apr. harvest cluster together in general, whereas the accessions from the 14 and 21 Apr. cluster more closely together. 'Baron Solemacher' is the only accession with harvest 1

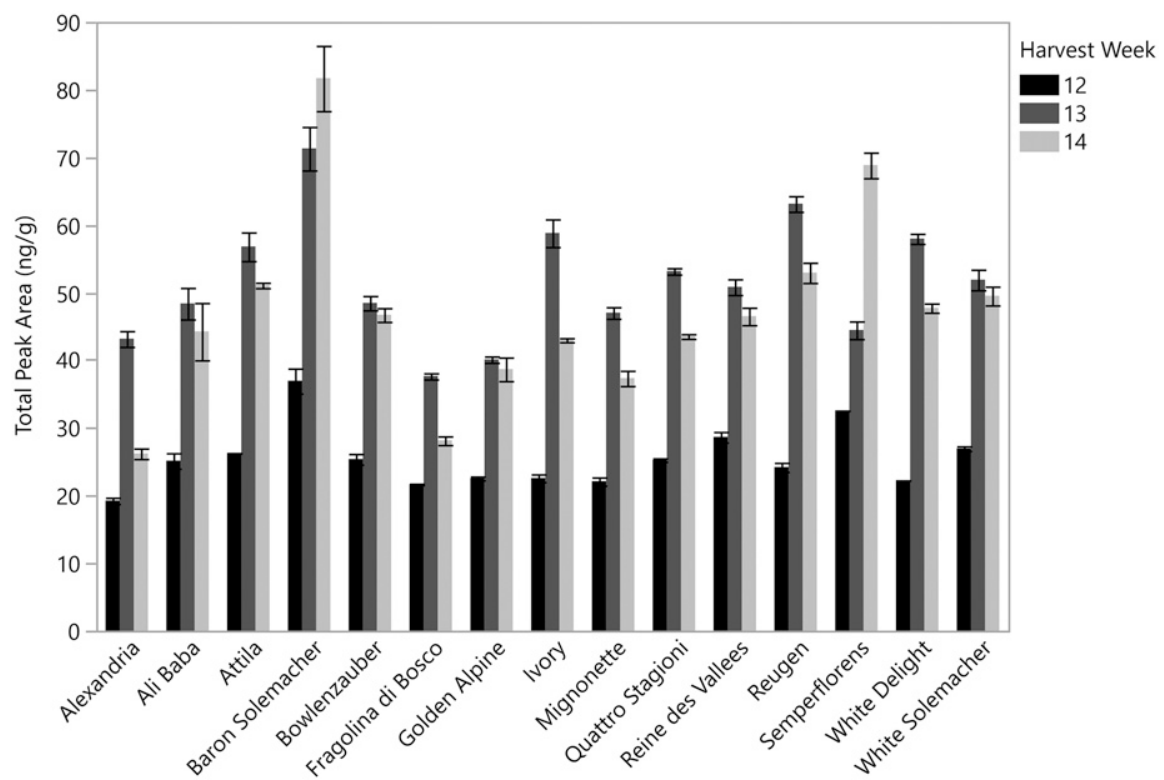

Fig. 3. Total peak area (ng/g) of 42 aroma compounds for each Fragaria vesca accession over three harvests on 7, 14, and 21 Apr. Data represent ripe fruit from three plots of 10 plants each bulked and analyzed by plot. Bars represent the SE of technical replicates. 
clustering with the majority of the accessions from harvests 2 and 3. Conversely, only harvest 3 for 'Alexandria' and 'Fragolina di Bosco' clustered more closely with the majority of the harvest 1 accessions.

\section{Discussion}

$F$. vesca is a novel fruit crop with potential for high economic returns. A compact growth habit and short time to harvest make this an attractive winter crop option, especially for growers in Florida, although other regions could easily cultivate this species. The soft fruit may not be suitable for extensive transport but could be an advantage for those targeting markets favoring local production or "u-pick" operations, where consumers pick their own produce on grower-managed land.

This is the first published study measuring yield and fruit quality for a large collection of $F$. vesca accessions. $F$. vesca yield data are lacking in general with the exception of previous work in Switzerland (four accessions, two locations) (Crespo et al., 2010), and hydroponic production of one $F$. vesca cultivar 'Regina delle Valli' (Caruso et al., 2011). Crespo et al. (2010) reported $F$. vesca yields from a single growing season between 173 and $661 \mathrm{~g}$ per plant, and Caruso et al. 2011 reported total yield at 185.2 and $128.2 \mathrm{~g}$ per plant for summer and autumn production cycles, respectively. During peak production in our study, the top accessions were yielding 13 to $14 \mathrm{~g}$ per plant per week, but total yield quantification is challenging to compare directly because the study lengths differ. Although there are many differences among these $F$. vesca yield trials, including cultivar selection and growing environment (soils, winter or summer production, nutrient management, pest management, etc.), they do contribute foundational data on the yield potential of currently existing $F$. vesca accessions.

TSS varied little among accessions and over multiple harvests demonstrating little diversity for this trait. The low correlations with fructose, glucose, sucrose, and total sugars demonstrates that TSS are probably not an acceptable proxy for sugar quantification in these accessions, in contrast to published results for commercial strawberry $(R=$ 0.74 TSS to total sugar content, Pistón et al., 2017). Alternatively, the narrow range of TSS data observed among all accessions in this study may not have necessary range to adequately test the correlation with sugar quantification. The overall levels of individual sugars reported in this study is similar to those reported in other $F$. vesca yield studies (Caruso et al., 2011; Crespo et al., 2010) and lower than commercial strawberries grown in Florida (Whitaker et al., 2011). Sweeter fruit in general is more desirable to consumers, and improving this trait in $F$. vesca will most likely require novel genetic solutions. Similar to total sugars, acidity is an important fruit quality trait. Acidity was measured in this study using an Atago optical sensor. One
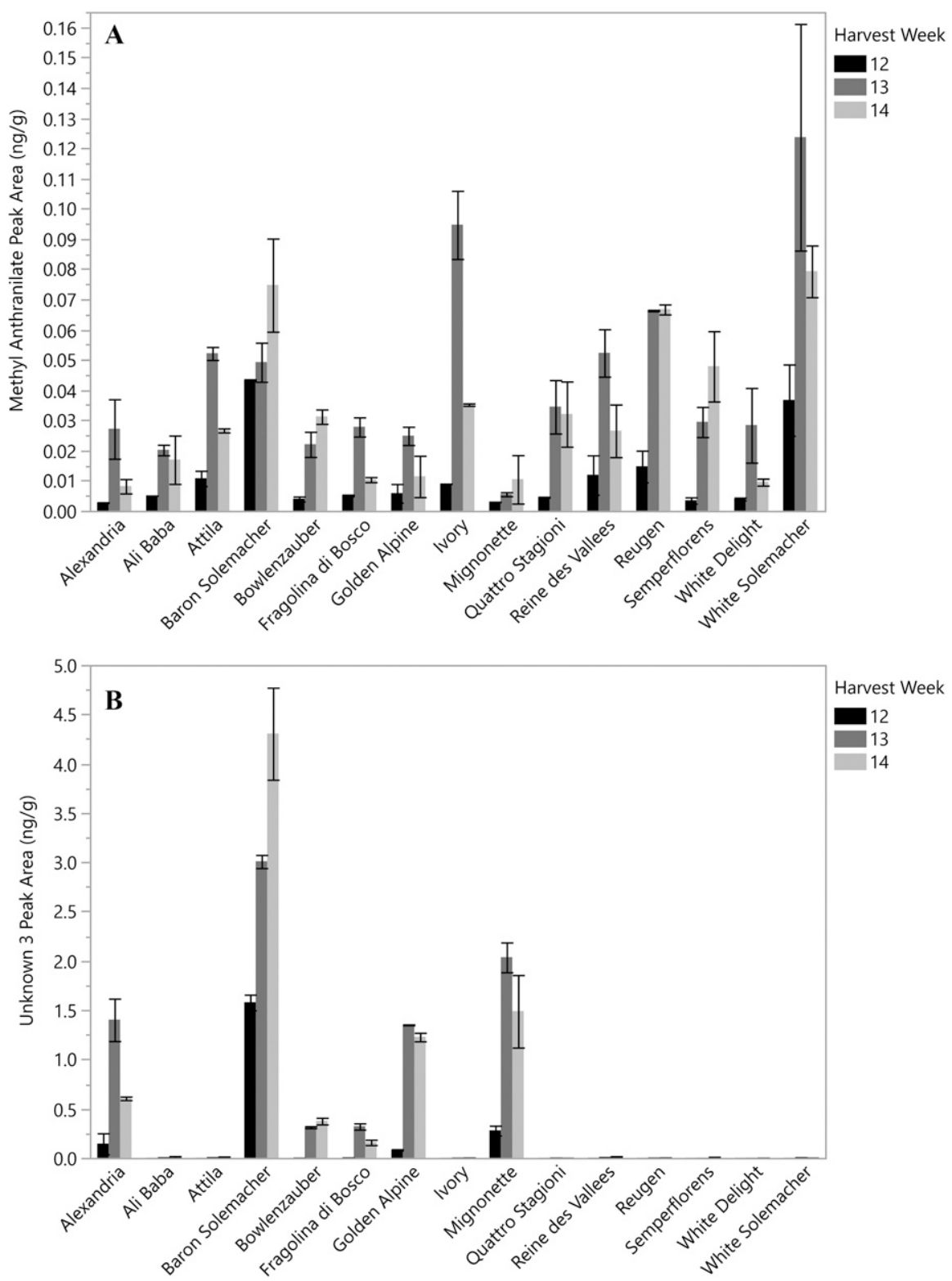

Fig. 4. Abundance (ng/g) of methyl anthranilate (A) and Unknown 3 (B) for each Fragaria vesca accession over three harvests (harvests 12,13, and 14 from 7, 14, and 21 Apr., respectively). Data represent ripe fruit from three plots of 10 plants each bulked and analyzed by plot. Bars represent the SE.

advantage of this sensor is the minimal extract volume required for analysis. This is especially useful for analyzing many accessions with limited sample sizes available for analytical analyses. Overall, there was little variability in acidity levels among the $F$. vesca accessions and over multiple harvests.

The total relative peak area from 42 semiquantitated aroma compounds varied more than 2-fold over multiple harvests during the growing season in Florida. The environmental impacts on aroma compounds have been reported for commercial strawberries in Florida (Chambers et al., 2014; Schwieterman et al., 2014). The impact of the Florida growing environment on aroma for multiple $F$. vesca accessions is now shown for the first time, but any potential effects on sensory quality are still unknown. The abundance of important aroma compounds such as methyl anthranilate (grape-like) appeared to fluctuate in $F$. vesca similarly to $F$. Xananassa (Pillet et al., 2017), although they were detectable in all $F$. vesca accessions tested unlike the majority of $F$. xananassa cultivars. Methyl anthranilate is believed to be an important aroma impact compound in $F$. vesca and is responsible for the typical "wood-strawberry" aroma (Ulrich et al., 2007).

Future genetic improvement of $F$. vesca will depend on the available diversity for key traits including yield and fruit quality. $F$. vesca is commonly propagated by seed, and selfing for many generations has led to a number of commercially available semiinbred lines. Differences among accessions were noticed during early season growth, including plant growth rate and plant architecture, and these traits were consistent 


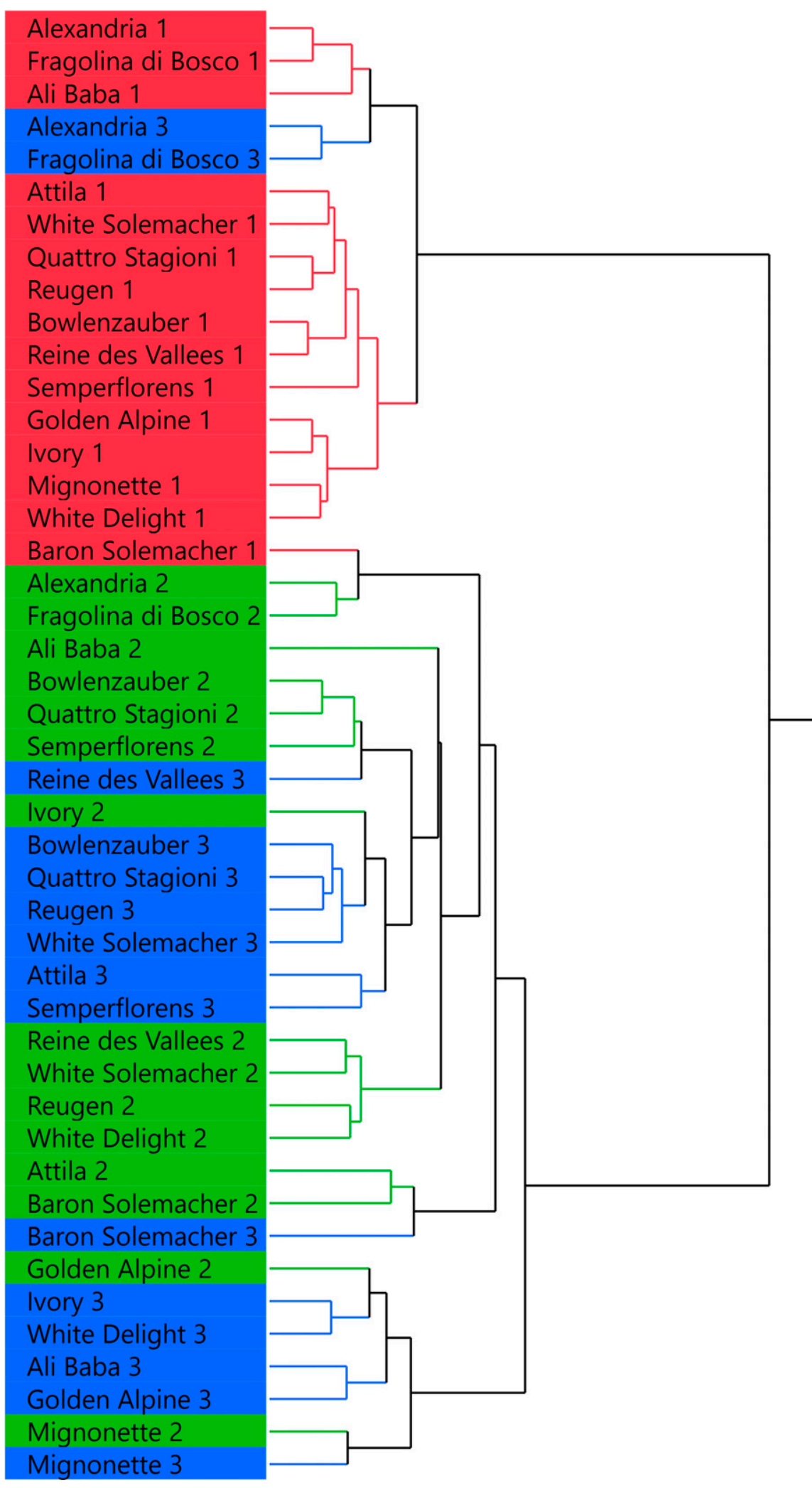

Fig. 5. Clustering of $F$. vesca accessions using data from 42 volatiles. Each accession was analyzed by three independent harvests noted with a number after the accessions name and color-coded (1, 2, and 3 represent harvest weeks 12, 13, and 14 on 7, 14, and 21 Apr., respectively). See online version for color figure.

within plots. Early season morphological differences became less distinct as development progressed to larger, fruiting plants.

This work demonstrates the superior yield performance of 'Reine des Vallees', 'Baron Solemacher', 'Fragolina di Bosco', 'Reugen', sugars, acidity, and aroma, suggest that the current $F$. vesca collection would benefit from increased diversity to improve cultivars through traditional breeding. Overall, these results support growers seeking new opportunities growing specialty fruits in southern Florida.

\section{Literature Cited}

Baldwin, E.A., J.H. Bai, A. Plotto, R. Cameron, G. Luzio, J. Narciso, J. Manthey, W. Widmer, and B.L. Ford. 2012. Effect of extraction method on quality of orange juice: Hand-squeezed, commercial-fresh squeezed and processed. J. Sci. Food Agr. 92:2029-2042.

Caruso, G., G. Villari, G. Melchionna, and S. Conti. 2011. Effects of cultural cycles and nutrient solutions on plant growth, yield and fruit quality of alpine strawberry (Fragaria vesca L.) grown in hydroponics. Scientia Hort. 129:479-485.

Chambers, A.H., J. Pillet, A. Plotto, J. Bai, V.M. Whitaker, and K.M. Folta. 2014. Identification of a strawberry flavor gene candidate using an integrated genetic-genomic-analytical chemistry approach. BMC Genomics 15:217.

Crespo, P., J.G. Bordonaba, L.A. Terry, and C. Carlen. 2010. Characterisation of major taste and health-related compounds of four strawberry genotypes grown at different Swiss production sites. Food Chem. 122:16-24.

Dong, J., Y.T. Zhang, X.W. Tang, W.M. Jin, and Z.H. Han. 2013. Differences in volatile ester composition between Fragaria $\times$ ananassa and $F$. vesca and implications for strawberry aroma patterns. Scientia Hort. 150:47-53.

Doumett, S., D. Fibbi, A. Cincinelli, E. Giordani, S. Nin, and M. Del Bubba. 2011. Comparison of nutritional and nutraceutical properties in cultivated fruits of Fragaria vesca L. produced in Italy. Food Res. Intl. 44:1209-1216.

Food and Agriculture Organization (FAO). 2016. Global fruit production in 2016 , by variety (in million metric tons)*. Statista. 19 June 2018 $<$ https://www.statista.com/statistics/264001/ worldwide-production-of-fruit-by-variety/>.

Giordani, E., C. Ancillotti, W.A. Petrucci, L. Ciofi, D. Morelli, C. Marinelli, L. Checchini, S. Furlanetto, and M. Del Bubba. 2016. Morphological, nutraceutical and sensorial properties of cultivated Fragaria vesca L. berries: Influence of genotype, plant age, fertilization treatment on the overall fruit quality. Agr. Food Sci. 25:187-201.

Hadonou, A.M., D.J. Sargent, F. Wilson, C.M. James, and D.W. Simpson. 2004. Development of microsatellite markers in Fragaria, their use in genetic diversity analysis, and their potential for genetic linkage mapping. Genome 47:429 438.

Hirakawa, H., K. Shirasawa, S. Kosugi, K. Tashiro, S. Nakayama, M. Yamada, M. Kohara, A. Watanabe, Y. Kishida, T. Fujishiro, H. Tsuruoka, C. Minami, S. Sasamoto, M. Kato, K. Nanri, A. Komaki, T. Yanagi, G.X. Qin, F. Maeda, M. Ishikawa, S. Kuhara, S. Sato, S. Tabata, and S.N. Isobe. 2014. Dissection of the octoploid strawberry genome by deep sequencing of the genomes of Fragaria species. DNA Res. $21: 169-181$

Jouquand, C., C. Chandler, A. Plotto, and K. Goodner. 2008. A sensory and chemical analysis of fresh strawberries over harvest dates and seasons reveals factors that affect eating quality. J. Amer. Soc. Hort. Sci. 133:859-867.

MacKenzie, S.J., C.K. Chandler, T. Hasing, and V.M. Whitaker. 2011. The role of temperature 
in the late-season decline in soluble solids content of strawberry fruit in a subtropical production system. HortScience 46:15621566.

Negri, A.S., D. Allegra, L. Simoni, F. Rusconi, C. Tonelli, L. Espen, and M. Galbiati. 2015. Comparative analysis of fruit aroma patterns in the domesticated wild strawberries "Profumata di Tortona" (F. moschata) and "Regina delle Valli" (F. vesca). Front. Plant Sci. $6: 56$.

Nin, S., W.A. Petrucci, E. Giordani, and C. Marinelli. 2017. Soilless systems as an alternative to wild strawberry (Fragaria vesca L.) traditional open-field cultivation in marginal lands of the Tuscan Apennines to enhance crop yield and producers' income. J. Hort. Sci. Biotechnol. 93:323-335.

Oosumi, T., H.A. Gruszewski, L.A. Blischak, A.J. Baxter, P.A. Wadl, J.L. Shuman, R.E. Veilleux, and V. Shulaev. 2006. High-efficiency transformation of the diploid strawberry (Fragaria vesca) for functional genomics. Planta 223:1219 1230.

Pillet, J., A.H. Chambers, C. Barbey, Z. Bao, A. Plotto, J. Bai, M. Schwieterman, T. Johnson, B. Harrison, V.M. Whitaker, T.A. Colquhoun, and K.M. Folta. 2017. Identification of a methyltransferase catalyzing the final step of methyl anthranilate synthesis in cultivated strawberry. BMC Plant Biol. 17:147.

Pistón, F., A.G. Pérez, C. Sanz, and A. Refoyo. 2017. Relationship between sugar content and
${ }^{\circ}$ Brix as influenced by cultivar and ripening stages of strawberry. Acta Hort. 1156:491-496.

Sargent, D.J., T.M. Davis, K.R. Tobutt, M.J. Wilkinson, N.H. Battey, and D.W. Simpson. 2004. A genetic linkage map of microsatellite, gene-specific and morphological markers in diploid Fragaria. Theor. Appl. Genet. 109:13851391.

Schwieterman, M.L., T.A. Colquhoun, E.A. Jaworski, L.M. Bartoshuk, J.L. Gilbert, D.M. Tieman, A.Z. Odabasi, H.R. Moskowitz, K.M. Folta, H.J. Klee, C.A. Sims, V.M. Whitaker, and D.G. Clark. 2014. Strawberry favor: Diverse chemical compositions, a seasonal influence, and effects on sensory perception. PLoS One 9: e88446.

Shulaev, V., D.J. Sargent, R.N. Crowhurst, T.C. Mockler, O. Folkerts, A.L. Delcher, P. Jaiswal, K. Mockaitis, A. Liston, S.P. Mane, P. Burns, T.M. Davis, J.P. Slovin, N. Bassil, R.P. Hellens, C. Evans, T. Harkins, C. Kodira, B. Desany, O.R. Crasta, R.V. Jensen, A.C. Allan, T.P. Michael, J.C. Setubal, J.M. Celton, D.J.G. Rees, K.P. Williams, S.H. Holt, J.J.R. Rojas, M. Chatterjee, B. Liu, H. Silva, L. Meisel, A. Adato, S.A. Filichkin, M. Troggio, R. Viola, T.L. Ashman, H. Wang, P. Dharmawardhana, J. Elser, R. Raja, H.D. Priest, D.W. Bryant, S.E. Fox, S.A. Givan, L.J. Wilhelm, S. Naithani, A. Christoffels, D.Y. Salama, J. Carter, E.L. Girona, A. Zdepski, W.Q. Wang, R.A. Kerstetter, W. Schwab, S.S. Korban, J. Davik, A. Monfort, B. Denoyes-Rothan, P. Arus, R. Mittler, B. Flinn,
A. Aharoni, J.L. Bennetzen, S.L. Salzberg, A.W. Dickerman, R. Velasco, M. Borodovsky, R.E. Veilleux, and K.M. Folta. 2011. The genome of woodland strawberry (Fragaria vesca). Nat. Genet. 43:109-116.

Ulrich, D., E. Hoberg, A. Rapp, and S. Kecke. 1997. Analysis of strawberry flavour - discrimination of aroma types by quantification of volatile compounds. Zeitschrift fur Lebensmittel Untersuching Forschung A 205:218-223.

Ulrich, D., D. Komes, K. Olbricht, and E. Hoberg. 2007. Diversity of aroma patterns in wild and cultivated Fragaria accessions. Genet. Resources Crop Evol. 54:1185-1196.

Ulrich, D. and K. Olbricht. 2013. Diversity of volatile patterns in sixteen Fragaria vesca L. accessions in comparison to cultivars of Fragaria $\times$ ananassa. J. Appl. Bot. Food Qual. 86:37-46.

Ulrich, D. and K. Olbricht. 2014. Diversity of metabolite patterns and sensory characters in wild and cultivated strawberries. J. Berry Res. 4:11-17.

U.S. Department of Agriculture. 2016. Strawberry production in the United States in 2016, by state (in 1,000 cwt)*. Statista. 19 June 2018. <https://www.statista.com/statistics/194235/ top-10-strawbery-producing-us-states/>.

Whitaker, V.M., T. Hasing, C.K. Chandler, A. Plotto, and E. Baldwin. 2011. Historical trends in strawberry fruit quality revealed by a trial of University of Florida cultivars and advanced selections. HortScience 46:553-557. 